Article

\title{
Enhanced Tensile Properties of Multi-Walled Carbon Nanotubes Filled Polyamide 6 Composites Based on Interface Modification and Reactive Extrusion
}

\author{
Min Park ${ }^{1,2}$, Ji-un Jang ${ }^{1}$, Jong Hyuk Park ${ }^{1}$, Jaesang $\mathrm{Yu}^{1, *}{ }^{1,0}$ and Seong Yun Kim ${ }^{3, *}$ \\ 1 Korea Institute of Science and Technology (KIST), Seoul 02792, Korea; minpark@kist.re.kr (M.P.); \\ jju204@naver.com (J.-u.J.); hyuk0326@kist.re.kr (J.H.P.) \\ 2 KHU-KIST Department of Converging Science and Technology, Kyung Hee University, Seoul 02447, Korea \\ 3 Division of Polymer-Nano \& Textile Engineering, Chonbuk National University, Jeonju-si 54896, Korea \\ * Correspondence: jamesyu@kist.re.kr (J.Y.); sykim82@jbnu.ac.kr (S.Y.K.)
}

Received: 1 April 2020; Accepted: 23 April 2020; Published: 25 April 2020

\begin{abstract}
To induce uniform dispersion and excellent interfacial properties, we adopted a strategy of combining both polyamide 6 (PA6) grafting for multi-walled carbon nanotubes (MWCNTs) and reactive extrusion of PA6 matrix, based on anionic ring-opening polymerization of $\varepsilon$-caprolactam (CL). Compared to $-\mathrm{COOH}$ and -NCO treatments of MWCNTs, enhanced MWCNT dispersion and tensile properties of the composites were achieved using the applied strategy, and the tensile strength and modulus of the PA6-grafted MWCNT-filled PA6 composites were $5.3 \%$ and $20.5 \%$ higher than those of the purified MWCNT-filled PA6 composites, respectively. In addition, they were almost similar to the theoretical ones calculated by the modified Mori-Tanaka method (MTM) assuming a perfect interface, indicating that the tensile properties of MWCNT-filled PA6 composites can be optimized by PA6 grafting and reactive extrusion based on the anionic ring-opening polymerization of CL due to uniform MWCNT dispersion and excellent interfacial property.
\end{abstract}

Keywords: polymer-matrix composites; carbon nanotubes; micro-mechanics

\section{Introduction}

Since their discovery [1], carbon nanotubes (CNTs) have been of great interest as an efficient reinforcement that can improve the mechanical properties of polymer composites due to their excellent mechanical properties such as 11-150 GPa tensile strength and 0.4-4.15 TPa tensile modulus [2-4]. However, there have been few reports on the successful development of CNT-based high strength polymer composites to meet researchers' expectations [5,6]. Uneven dispersion or aggregation of CNTs in a composite material due to van der Waals force has been pointed out as one of the obstacles to the development of high strength composite material using CNTs $[4,7,8]$. In addition, it is difficult to induce interfacial bonding between nano-sized CNTs and polymers based on a mechanical interlocking mechanism, so they exhibit limited interfacial properties $[4,9,10]$. In order to overcome these problems, proper CNT surface treatment is required in consideration of the characteristics of the polymer matrix and the manufacturing process of composite materials.

Appropriate CNT surface treatment not only improves the dispersion of CNTs in composites but also maximizes the interfacial properties between CNTs and polymeric matrices via mechanisms such as hydrogen bonding, covalent and noncovalent bonding, and polymer interdiffusion [11-13]. CNTs can be treated with an acid to introduce $-\mathrm{COOH}$ groups, which can induce a hydrogen bond or a covalent bond with the polyamide 6 (PA6) matrix $[14,15]$. The interfacial bonding between CNTs and PA6 matrix can also be improved by introducing -NCO groups on the surfaces of CNTs $[16,17]$. A previous study [18] reported that PA6 polymers can be grafted on the surface of CNTs, and PA6 
polymers on the surface of CNTs are interdiffused with PA6 matrix, resulting in excellent dispersion as well as improved interfacial properties $[7,16,19,20]$.

The manufacturing process of composite materials filled with CNTs can be classified into three main types based on the dispersion method: melt mixing, solution mixing, and in-situ polymerization [4, 6,21-23]. The melt mixing method is the most economical but is difficult to induce sufficient dispersion. Solution mixing makes it easy to generate uniform dispersion, but it is not economical due to the use of a solvent and the long drying process. For this reason, an in-situ polymerization process based on an economical melting process can be selected, while inducing sufficient dispersion. In particular, it has been widely reported that the reactive polymerization of thermoplastic matrix can induce excellent dispersion of nanofillers, thereby substantially improving the bulk properties of the composites filled with the well-dispersed nanofillers [18-30].

Although reactive extrusion using anionic polymerization of $\varepsilon$-caprolactam (CL) is an appealing composite process for nanofiller dispersion in terms of both the cost of raw materials and the characteristics of matrix materials, there have been few systematic studies simultaneously applying both reactive extrusion based on the anionic polymerization of $\mathrm{CL}$ and the various surface treatments of multi-walled carbon nanotubes (MWCNTs). In this study, $-\mathrm{COOH},-\mathrm{NCO}$, and -PA6 functionalized MWCNTs were prepared, and PA6 composites filled with the surface treated MWCNTs were fabricated by the reactive extrusion based on the anionic polymerization of CL. MWCNT dispersion and tensile properties of the composites were evaluated in order to investigate the effects of the MWCNT surface treatments and the reactive extrusion. By comparing between the measured tensile properties and the theoretically calculated ones based on the modified Mori-Tanaka method (MTM), interfacial properties of the composites were evaluated.

\section{Experimental}

\subsection{Materials}

MWCNTs supplied from the Jeio Co. (Inchon, Korea) were used as a reinforcement. Pristine MWCNTs were purified by sonication in $3 \mathrm{M} \mathrm{HCl}$ solution for $6 \mathrm{~h}$ and then filtered using a polytetrafluoroethylene (PTFE) filter. The purified MWCNTs were washed with distilled water until reaching $\mathrm{pH} 7$, then vacuum-dried at $80^{\circ} \mathrm{C}$. CL supplied by Capro (Seoul, Korea) was used as a matrix. Sodium hydride (NaH, Sigma-Aldrich, Yongin, Korea) and hexamethylene diisocyanate (HDI, Sigma-Aldrich, Yongin, Korea) were used as a catalyst and activator, respectively, for anionic polymerization of CL. The mechanism of anionic polymerization of CL has been widely investigated and reported as shown in Figure S1 [16].

\subsection{Surface Treatment of MWCNT}

\subsubsection{Carboxylated MWCNT}

First, $1 \mathrm{wt} \%$ purified MWCNT was added to the mixed solution of $\mathrm{HNO}_{3} / \mathrm{H}_{2} \mathrm{SO}_{4}$ at a ratio of 1:3. The mixture was sonicated at $80^{\circ} \mathrm{C}$ for $6 \mathrm{~h}$ and then filtered using a PTFE filter. The prepared MWCNT was rinsed with distilled water until reaching $\mathrm{pH} 7$, then vacuum-dried at $80^{\circ} \mathrm{C}$ to prepare carboxylated MWCNT.

\subsubsection{Isocyanated MWCNT}

First, $1 \mathrm{wt} \%$ carboxylated MWCNT was sonicated in anhydrous acetone, and then $4 \mathrm{vol} \% \mathrm{HDI}$ was added. The reaction was induced by sonication under dry $\mathrm{N}_{2}$ gas at $50^{\circ} \mathrm{C}$ for $24 \mathrm{~h}$, then filtered using a PTFE filter. 


\subsubsection{PA6 Grafted MWCNT}

Isocyanated MWCNTs were mixed with molten $\mathrm{CL}$, sonicated at $110^{\circ} \mathrm{C}$ for $6 \mathrm{~h}$, and then mixed with molten CL containing NaH catalyst. The CL was propagated from the MWCNT surface, resulting in a PA6-grafted MWCNT in which the MWCNT and PA6 were covalently linked.

\subsection{Composite Fabrication}

The $\mathrm{NaH}$ was added to the $\mathrm{CL}$ melt, which was removed to a water content of $50 \mathrm{ppm}$ or less. The reaction proceeded at $110{ }^{\circ} \mathrm{C}$ until the hydrogen gas generation was completed, and then the air was sealed. Then, the HDI was added to the CL melt, which was removed to a water content of $50 \mathrm{ppm}$ or less. After reacting at $110^{\circ} \mathrm{C}$ for $1 \mathrm{~h}$, it was sealed with the air removed. The prepared mixture was reactive extruded using a twin-screw extruder (L40/D19, Bautek CO, Uijungbu, Korea) under optimized process conditions that were derived as shown in Figure S2. At this time, the residence time of the mixture in the extruder was $100 \mathrm{~s}$. To determine the optimum reaction temperature and concentration which would set the reaction completion time to be shorter than the residence time of the mixture, anionic polymerization of the CL proceeded under various conditions. To investigate the effect of MWCNT surface treatment on the structural and tensile characteristics of composites, $1 \mathrm{wt} \%$ purified, carboxylated, isocyanated, and PA6-grafted MWCNTs were dispersed in the activator mixture and then mixed with the catalyst mixture. In the reactive extrusion process, PA6 composites were prepared by the anionic polymerization of the CL mixture matrix as shown in Figure 1.

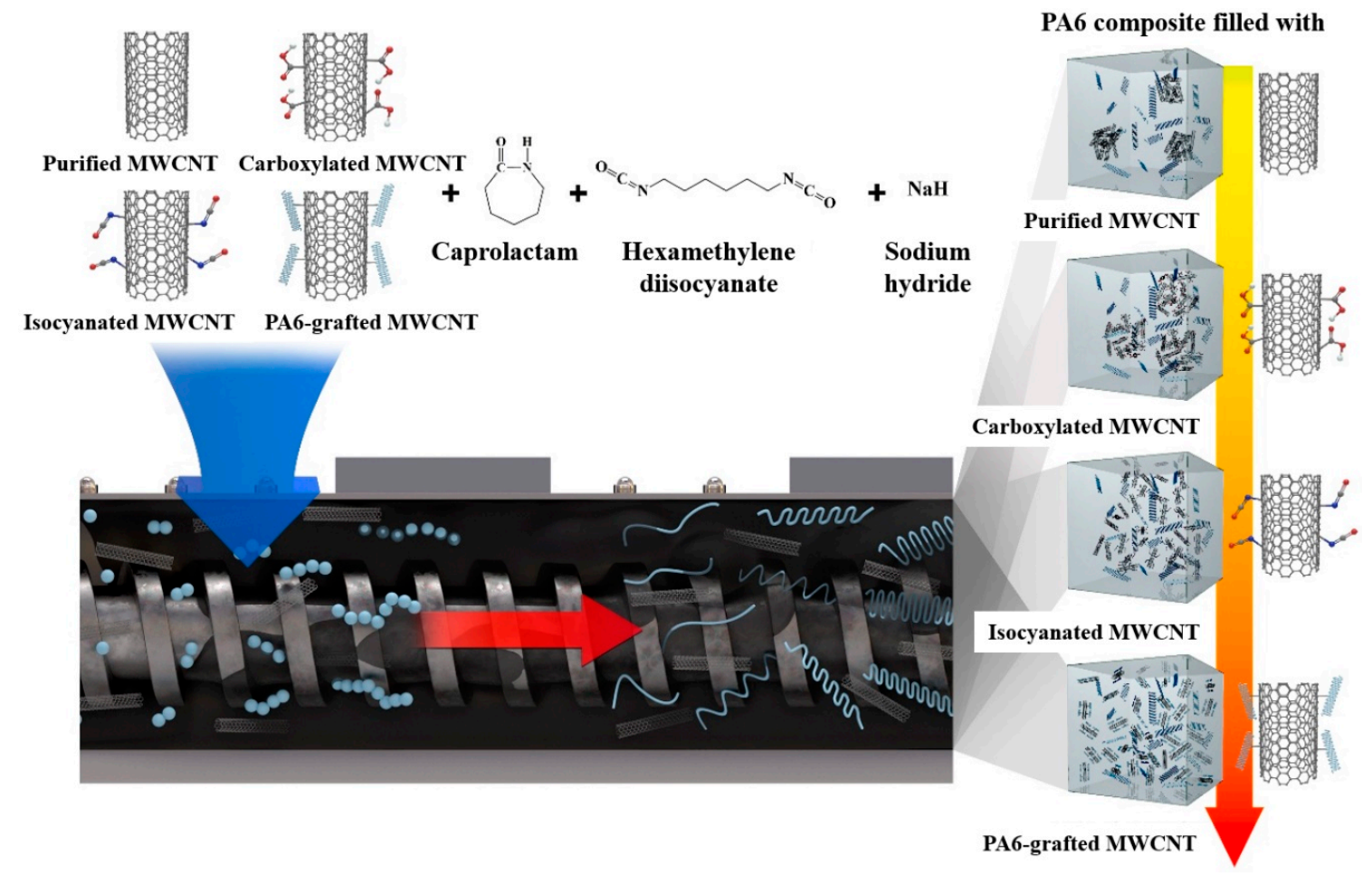

Figure 1. Schematic of the applied strategy.

\subsection{Characterization}

Fourier transform infrared spectroscopy (FTIR, Nicolet 6700, Thermo Scientific, Waltham MA, USA) analysis was performed to observe the chemical changes of MWCNTs by the surface treatments. The FTIR spectrum was measured at $8 \mathrm{~cm}^{-1}$ resolution in the wave number range of $500-4000 \mathrm{~cm}^{-1}$. To observe the compatibility between the prepared MWCNTs and PA6 matrix, the precipitation of MWCNTs in the formic acid, a good solvent of PA6, was observed for 20 days after sonication. The structural changes of MWCNTs were observed by the surface treatments using transmission 
electron microscopy (TEM, Tecnai F20, FEI Corp., Hillsboro OR, USA). In order to prepare the TEM samples, the purified, carboxylated, isocyanated, and PA6-grafted MWCNTs were dispersed in ethanol and sonicated for $5 \mathrm{~min}$, then the dispersion was dropped on the lacey-carbon coated film on the $\mathrm{Cu}$ grid. TEM observations were performed at $120 \mathrm{kV}$, and the exposure time of accelerating electrons was minimized in an attempt to avoid electron exposure damage during TEM analysis. The molecular weights of the PA6 and composite samples were determined using gel permeation chromatography (GPC, EcoSEC, Tosoh Bioscience, Tokyo, Japan). Samples dissolved in hexafluoroisopropanol with trifluoroacetic acid Na salt were left for one day and filtered with a $0.45 \mu \mathrm{m}$ PTFE filter. The GPC system was calibrated using polymethyl methacrylate standard. To evaluate the MWCNT dispersion, the fractured surface of the prepared composite was observed using a field emission scanning electron microscope (FE-SEM, Nova NanoSEM 450, FEI Corp., Hillsboro OR, USA) at a voltage of $15 \mathrm{kV}$ after coating $(\mathrm{Pt})$ under vacuum for $80 \mathrm{~s}$ by a sputter machine (Ion Sputter E-1030, Hitachi High Technologies, Tokyo, Japan). The tensile test was carried out using a universal tester (Instron 5567, Norwood, MA, USA) with a crosshead speed of $5 \mathrm{~mm} / \mathrm{min}$ at room temperature according to American society for testing and materials (ASTM) D638.

\section{Micromechanics Modeling Approach}

The Mori-Tanaka micromechanical approach has mainly been used to estimate effective heterogeneous material properties, particularly for composites containing low volume fractions of reinforcements in elastic resins. Yu et al. [31] developed a modified MTM for predicting effective elastic properties for nanocomposites containing multiple nano heterogeneities with arbitrary shapes and orientations.

\subsection{Modified Mori-Tanaka Method for Composite Stiffness Properties}

This approach may be extended to the case for composites containing multiple distinct heterogeneities. Suppose that the matrix contains $m$ distinct types of ellipsoidal heterogeneities $(k=1,2, \ldots, m)$, each consisting of $n_{k}$ layers $\left(\alpha_{k}=1,2, \ldots, n_{k} ; \mathrm{k}=1,2, \ldots, m\right)$. Each type of heterogeneity has distinct elastic properties, shape, and orientation distribution. Based on a composite containing $\mathrm{m}$ distinct types of heterogeneities $(k=1,2, \ldots, m)$, each with an arbitrary number of layers $\left(n_{k}\right)$ in a matrix (0), the overall elasticity tensor, $\bar{L}$, may be expressed as

$$
\begin{gathered}
\overline{\boldsymbol{L}}=\boldsymbol{L}^{(0)}:\left\{\boldsymbol{I}+\sum_{k=1}^{m}\left[\sum_{\alpha_{k}=1}^{n_{k}} \boldsymbol{c}_{(k)} \alpha_{k}\left(\boldsymbol{S}_{(k)}-\boldsymbol{I}\right):\left(\boldsymbol{A}_{(k)}\left(\alpha_{k}\right)-\boldsymbol{S}_{(k)}\right)^{-1}\right]\right\}: \\
\left\{\boldsymbol{I}+\sum_{k=1}^{m}\left[\sum_{\alpha_{k}=1}^{n_{k}} c_{(k)} \alpha_{k} \boldsymbol{S}_{(k)}:\left(\boldsymbol{A}_{(k)}\left(\alpha_{k}\right)-\boldsymbol{S}_{(k)}\right)^{-1}\right]\right\}
\end{gathered}
$$

where

$$
\boldsymbol{A}_{(k)}{ }^{\left(\alpha_{k}\right)}=\left(\boldsymbol{L}^{(0)}-\boldsymbol{L}_{(k)}\left(\alpha_{k}\right)\right)^{-1}: \boldsymbol{L}^{(0)}
$$

$\boldsymbol{A}_{(k)}\left(\alpha_{k}\right)$ is the local strain concentration tensor for the $\alpha_{k}{ }^{\text {th }}$ layer of the $k^{\text {th }}$ heterogeneity $\left(\alpha_{k}=1\right.$, $\left.2, \ldots, n_{k}, k=1,2, \ldots, m\right)$. Here, $\boldsymbol{L}_{(k)}\left(\alpha_{k}\right)$ is the fourth rank elastic stiffness tensor for the $\alpha_{k}{ }^{\text {th }}$ layer of the $k^{\text {th }}$ heterogeneity, $\mathcal{c}_{(k)} \alpha_{k}$ is the volume fraction of the $\alpha_{k}^{\text {th }}$ layer of the $k^{\text {th }}$ heterogeneity, and $S_{(k)}$ is the $4^{\text {th }}$ rank Eshelby tensor common to the heterogeneity and all layers of the $k^{\text {th }}$ heterogeneity.

\subsection{Modified Mori-Tanaka Method for Composite Strength Properties}

The ultimate applied stresses in heterogeneities and the matrix, $\left(\sigma_{\mathrm{A}} \mathrm{M}, \sigma_{\mathrm{A}} \Omega_{\alpha}\right)$, can be determined by the average stress reaching the strength properties of the matrix or the heterogeneities [32]. The composite strength property is dominated by the heterogeneity strength value when the ultimate applied stress in heterogeneities $\left(\sigma_{A} \Omega_{\alpha}\right)$ is less than that of the matrix $\left(\sigma_{A} \mathrm{M}\right)$. By contrast, the composite strength property is dominated by the matrix strength value when the ultimate applied stress in matrix 
$\left(\sigma_{\mathrm{A}}^{\mathrm{M}}\right)$ is less than that of heterogeneities $\left(\sigma_{\mathrm{A}} \Omega_{\alpha}\right)$. The ultimate applied stress $\left(\sigma_{\mathrm{A}}^{\mathrm{M}}\right)$ can be determined by Equation (3) when the average stress in the matrix, $\left(\sigma_{A}+\langle\sigma\rangle_{\mathrm{M}}\right)$, reaches the strength value of matrix $\left(\sigma_{s}{ }^{\mathrm{M}}\right)$; it can be expressed as follows:

$$
\boldsymbol{\sigma}_{\mathrm{A}}^{\mathrm{M}}=\left[\boldsymbol{I}-\sum_{\alpha=1}^{n} \mathrm{c}_{\alpha} \boldsymbol{L}^{(0)}:\left(\sum_{\alpha=1}^{n} \boldsymbol{S}^{(\alpha)}-\boldsymbol{I}\right): \sum_{\alpha=1}^{n} \alpha_{\alpha}^{-1}:\left(\boldsymbol{L}^{(0)}-\sum_{\alpha=1}^{n} \boldsymbol{L}^{(\alpha)}\right): \boldsymbol{L}^{(0)-1}\right]^{-1}: \boldsymbol{\sigma}_{s}^{\mathrm{M}}
$$

Similarly, the ultimate applied stress $\left(\sigma_{\mathrm{A}} \Omega_{\alpha}\right)$ can be determined by Equation (4), where the average stress in the $\boldsymbol{\alpha}^{\text {th }}$ heterogeneity $\left(\sigma_{A}+\sum_{\alpha=1}^{n}\langle\boldsymbol{\sigma}\rangle_{\Omega_{\alpha}}\right)$ reaches the strength value of $\boldsymbol{\alpha}^{\text {th }}$ heterogeneity $\left(\sigma_{s}^{\Omega_{\alpha}}\right)$; it can be expressed as follows:

$$
\boldsymbol{\sigma}_{\mathrm{A}} \Omega_{\alpha}=\left[\left(\boldsymbol{1}-\sum_{\alpha=1}^{n} c_{\alpha}\right) \boldsymbol{L}^{(0)}:\left(\sum_{\alpha=1}^{n} \boldsymbol{S}^{(\alpha)}-\boldsymbol{I}\right): \sum_{\alpha=1}^{n} \alpha_{\alpha}{ }^{-1}:\left(\boldsymbol{L}^{(0)}-\sum_{\alpha=1}^{n} \boldsymbol{L}^{(\alpha)}\right): \boldsymbol{L}^{(0)-1}+\boldsymbol{I}\right]^{-1}: \boldsymbol{\sigma}_{s}{ }^{\Omega_{\alpha}}
$$

\section{Results and Discussion}

\subsection{Surface Treatment of MWCNTs}

FTIR spectra are shown in Figure 2a to observe the surface functionality of MWCNTs by the applied surface treatments. The FTIR spectrum of the carboxylated MWCNTs showed O-H peaks near 3421 and $1453 \mathrm{~cm}^{-1}$, as well as peaks at 1654 and $1710 \mathrm{~cm}^{-1}$ due to the $\mathrm{C}=\mathrm{O}$ of carboxyl groups [16,17]. In the spectrum of the isocyanated MWCNT, the characteristic peak of $\mathrm{O}-\mathrm{H}$ disappeared, and a new peak of $2283 \mathrm{~cm}^{-1}$ was found, which corresponds to the asymmetric elongation of the isocyanate group [16]. The peaks at 2900 and $2825 \mathrm{~cm}^{-1}$ were due to the $\mathrm{C}-\mathrm{H}$ stretching of the methyl group of the diisocyanate [16]. The peak at $1245 \mathrm{~cm}^{-1}$ was attributed to the $\mathrm{C}-\mathrm{N}$ stretch of the carbamate group resulting from the esterification reaction of the hydroxyl group and the isocyanate group [16]. As compared with the FTIR spectrum of the isocyanated MWCNT, the disappearance of the peak at $2283 \mathrm{~cm}^{-1}$ in the FTIR spectrum of the PA6-grafted MWCNT indicated that the isocyanate group was consumed during the PA6 polymers grafting reaction [16]. In addition, the characteristic absorption peaks of PA6, such as 3297 (NH stretching), 3060, 1637 (amide I band), and $1540 \mathrm{~cm}^{-1}$ (amide II band) were observed [16]. Based on the FTIR results, it was confirmed that the carboxyl group was introduced into the MWCNT surface by acid treatment, the isocyanated MWCNT was prepared by the isocyanate treatment, and the activation of the isocyanate group for the anionic polymerization of CL resulted in the PA6-grafted MWCNT.

TEM images are shown in Figure 3 to observe the structural changes in MWCNTs by the surface treatments. Unlike the other MWCNTs, the PA6-grafted MWCNT was coated with about $8 \mathrm{~nm}$ of PA6 layer. In the case of the PA6-grafted MWCNT, the surface of MWCNT was covalently bonded to PA6 polymers, so it can be expected to exhibit improved compatibility with the PA6 matrix. Excellent compatibility between the PA6-grafted MWCNTs and PA6 matrix was observed based on the fact that no precipitation was observed only in the PA6-grafted MWCNT sample as shown in Figure 2b. 


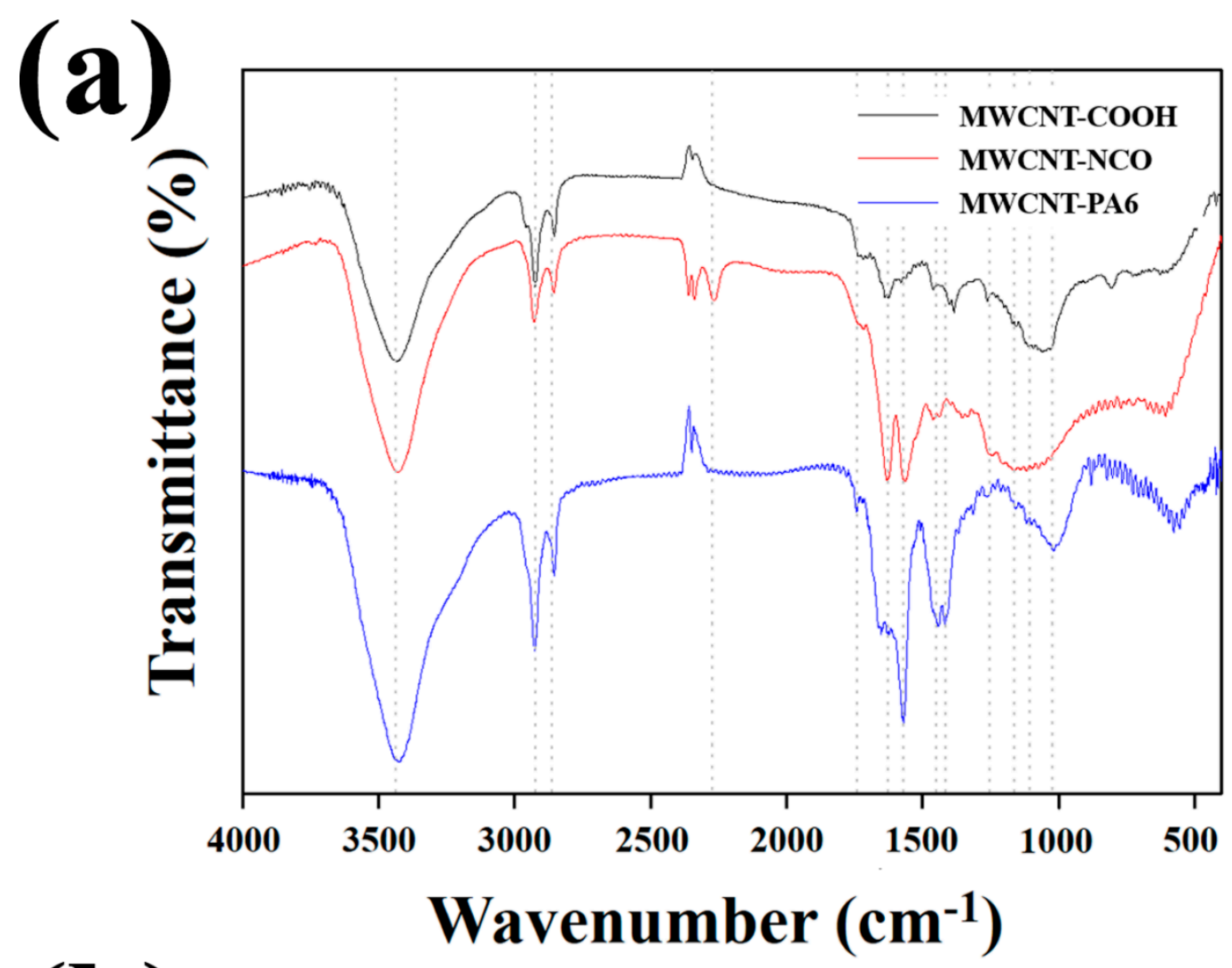

(b)
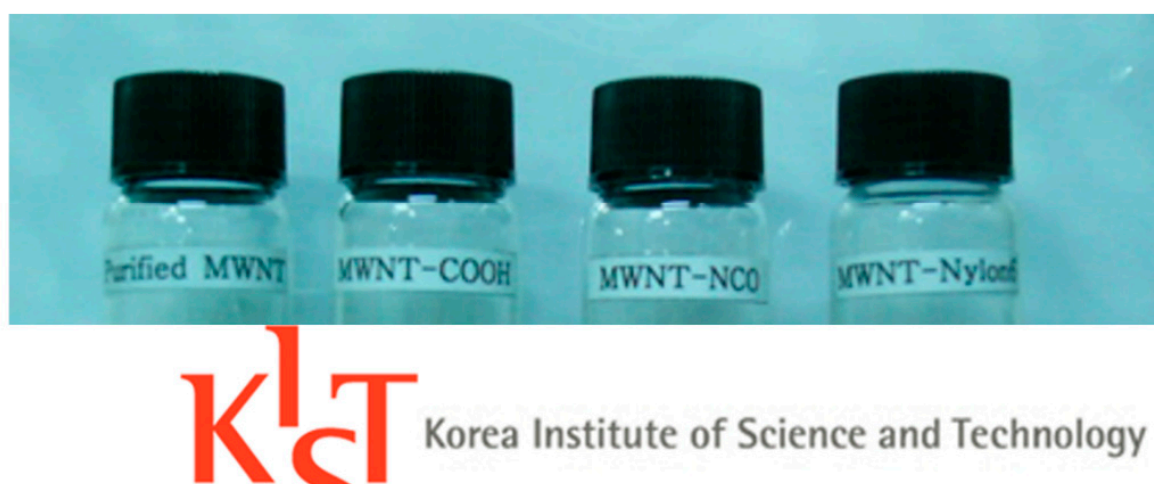

Korea Institute of Science and Technology

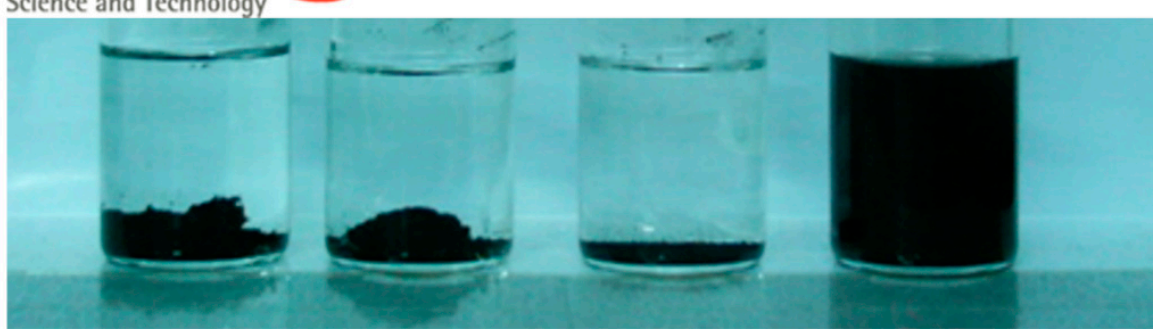

Figure 2. (a) Fourier transform infrared spectroscopy (FTIR) spectra of surface-treated multi-walled carbon nanotubes (MWCNTs) and (b) MWCNT dispersion in formic acid after 20 days after ultrasonication according to the surface treatment. 


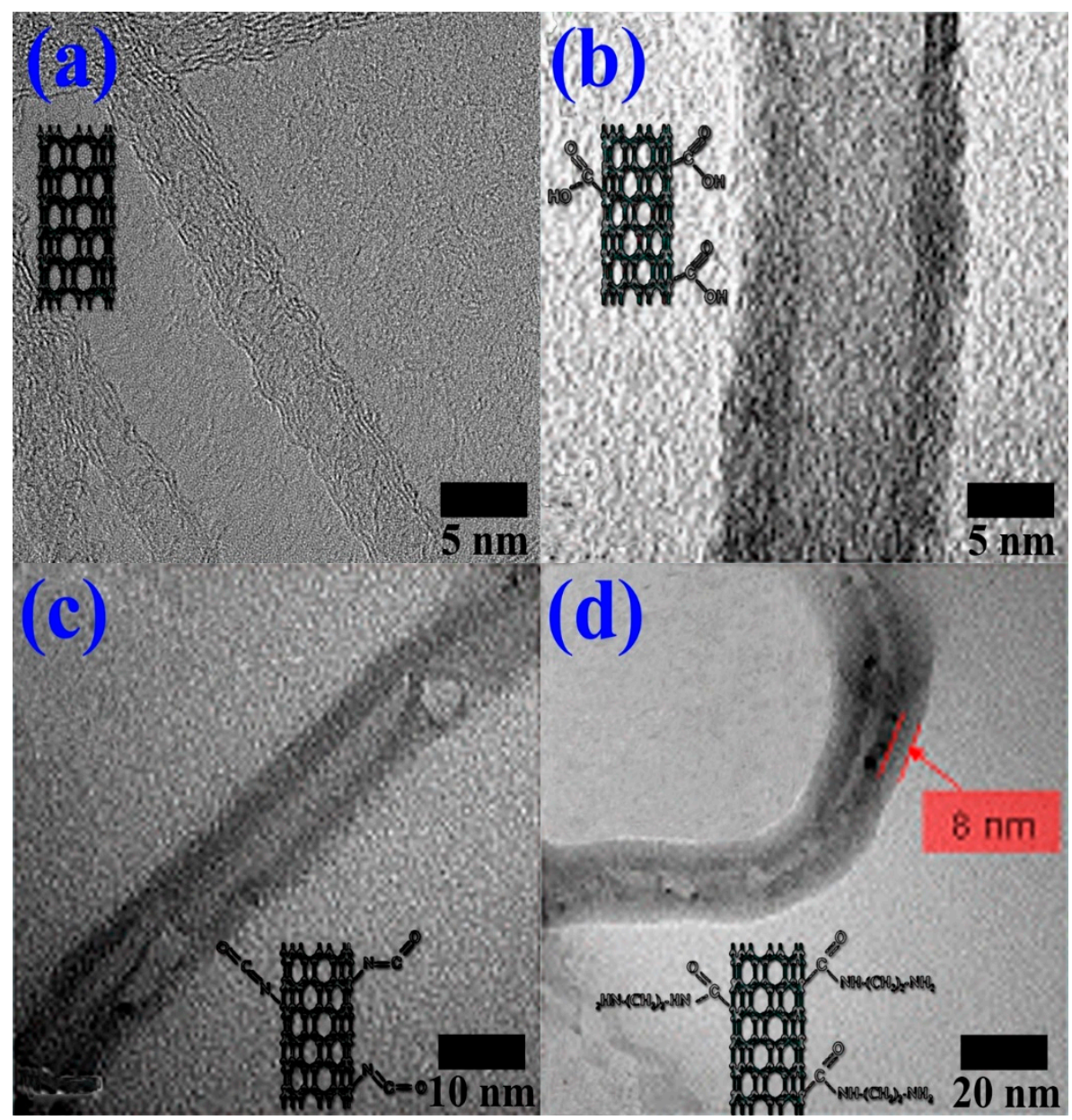

Figure 3. Transmission electron microscopy (TEM) images of (a) purified MWCNTs, (b) carboxylated MWCNTs, (c) isocyanated MWCNTs, and (d) polyamide 6 (PA6) grafted MWCNTs.

\subsection{Reactive Extrusion}

To complete the anionic polymerization of CL during the reactive extrusion process as shown in Figure 1, the reaction completion time must be faster than the residence time in the extruder. The rate of anionic polymerization of $\mathrm{CL}$ is known to be a function of reaction temperature as well as catalyst and activator concentrations $[18,33,34]$. To set an activator/catalyst ratio that can complete the anionic polymerization faster than the residence time of $100 \mathrm{~s}$, the optimized reactive extrusion process conditions are presented in Figure S2; Figure 4a shows the solidification time according to the activator/catalyst ratio. It was confirmed that the appropriate reaction completion time was induced when the ratio of activator in the mixture was 0.4 or $0.6 \mathrm{~mol} \%$ and the activator/catalyst ratio was higher than 1.5. Since the activator and especially the catalyst are more expensive than $\mathrm{CL}$, the optimum composition of $0.4 \mathrm{~mol} \%$ activator and $0.6 \mathrm{~mol} \%$ catalyst, which can minimize the use of these materials, was selected. Based on the optimum activator/catalyst composition as well as the reaction kinetics expression of Wittmer and Gerrens [35] as shown in Figure $4 \mathrm{~b}$ (details in Supplementary Materials), the optimum reaction temperature was determined. The higher the reaction temperature, the shorter the reaction time. However, at temperatures exceeding $240{ }^{\circ} \mathrm{C}$, there is a risk of ignition and thermal deformation due to the rapid reaction, and $240{ }^{\circ} \mathrm{C}$ can be considered as the optimum reaction temperature. 
(a)
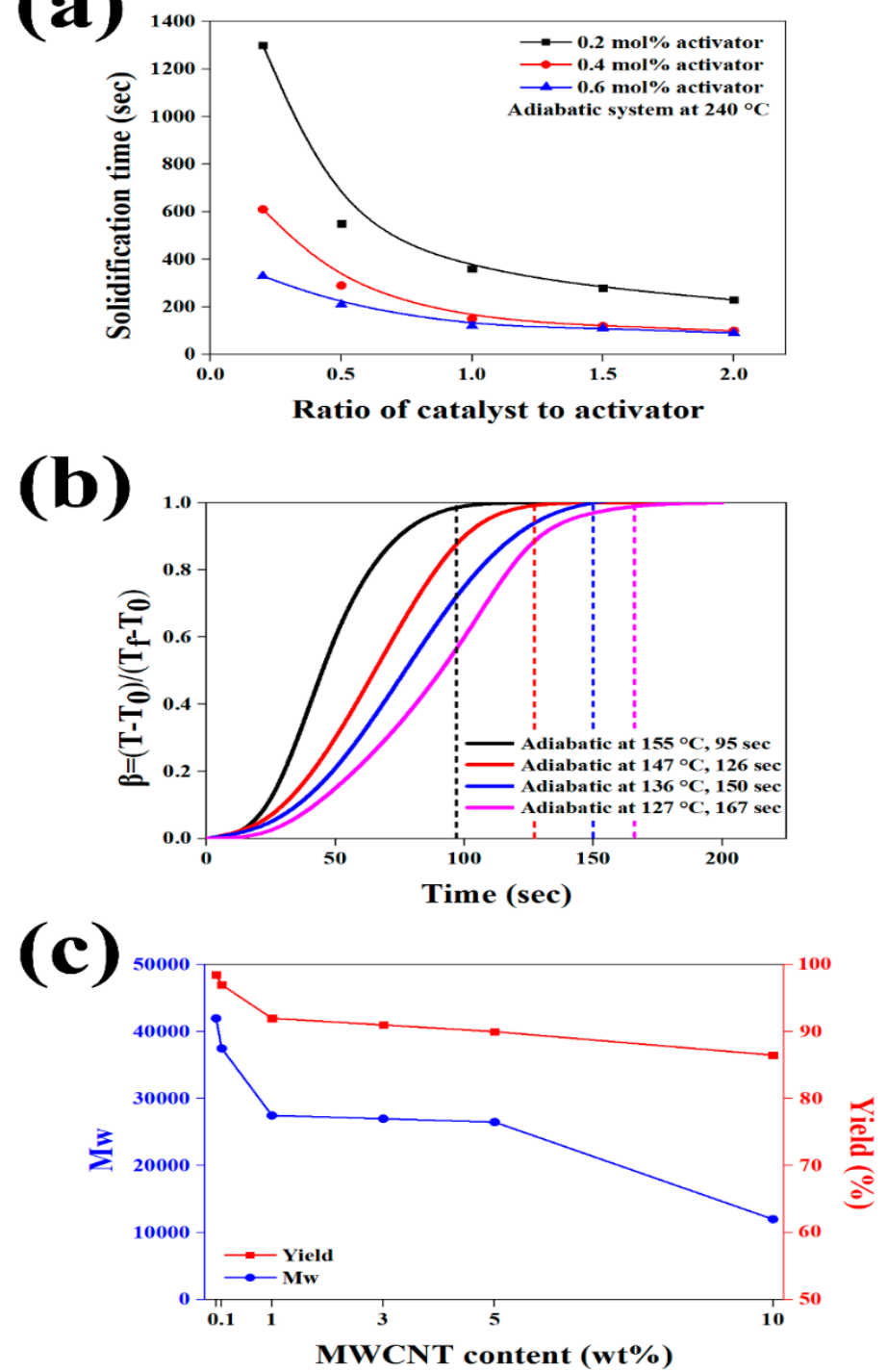

Figure 4. (a) Dependence of solidification time on the catalyst to activator ratio at $240{ }^{\circ} \mathrm{C},(\mathbf{b})$ reaction kinetics expressions of Wittmer and Gerrens, and (c) molecular weight and yield of polymerized PA6 according to MWCNT content.

In order to investigate the effect of MWCNT incorporation on the anionic polymerization, the molecular weight of the reactive extruded PA6 matrix is shown in Figure 4c according to the MWCNT content. The reactive extruded PA6 without added MWCNT showed a molecular weight of about 42,000 and a yield of $98 \%$. As the MWCNT content increases, the molecular weight and yield decreased because the mobility of the grown polymer chain was reduced due to the spatial restriction of the MWCNT [36]. When the MWCNT was incorporated within $5 \mathrm{wt} \%$ in the optimum process conditions, the molecular weight of the PA6 polymer exceeded 22,000, indicating good mechanical properties [37]. In addition, the effect of MWCNT functional groups on the molecular weight was insignificant, and similar molecular weight and yield were observed. Therefore, when the MWCNT was incorporated within $5 \mathrm{wt} \%$, a molecular weight higher than 22,000, which exhibited the good mechanical properties of PA6 matrix, was induced irrespective of the surface treatment of the MWCNT.

\subsection{MWCNT Dispersion of Composites}

Figure 5 shows the FE-SEM images of the fracture surface of the composites to evaluate the MWCNT dispersion in the PA6 composites produced by the reactive extrusion. In the fracture 
morphology images, the anionic polymerization of CL derived during the reactive extrusion process resulted in a quite uniform dispersion of MWCNTs. However, certain MWCNT agglomerates were found in the fracture surface of the composites containing the purified MWCNTs. In addition, MWCNT dispersion was further improved by applying the surface treatments. In particular, when PA6-grafted MWCNTs were applied, the uniform dispersion was confirmed.

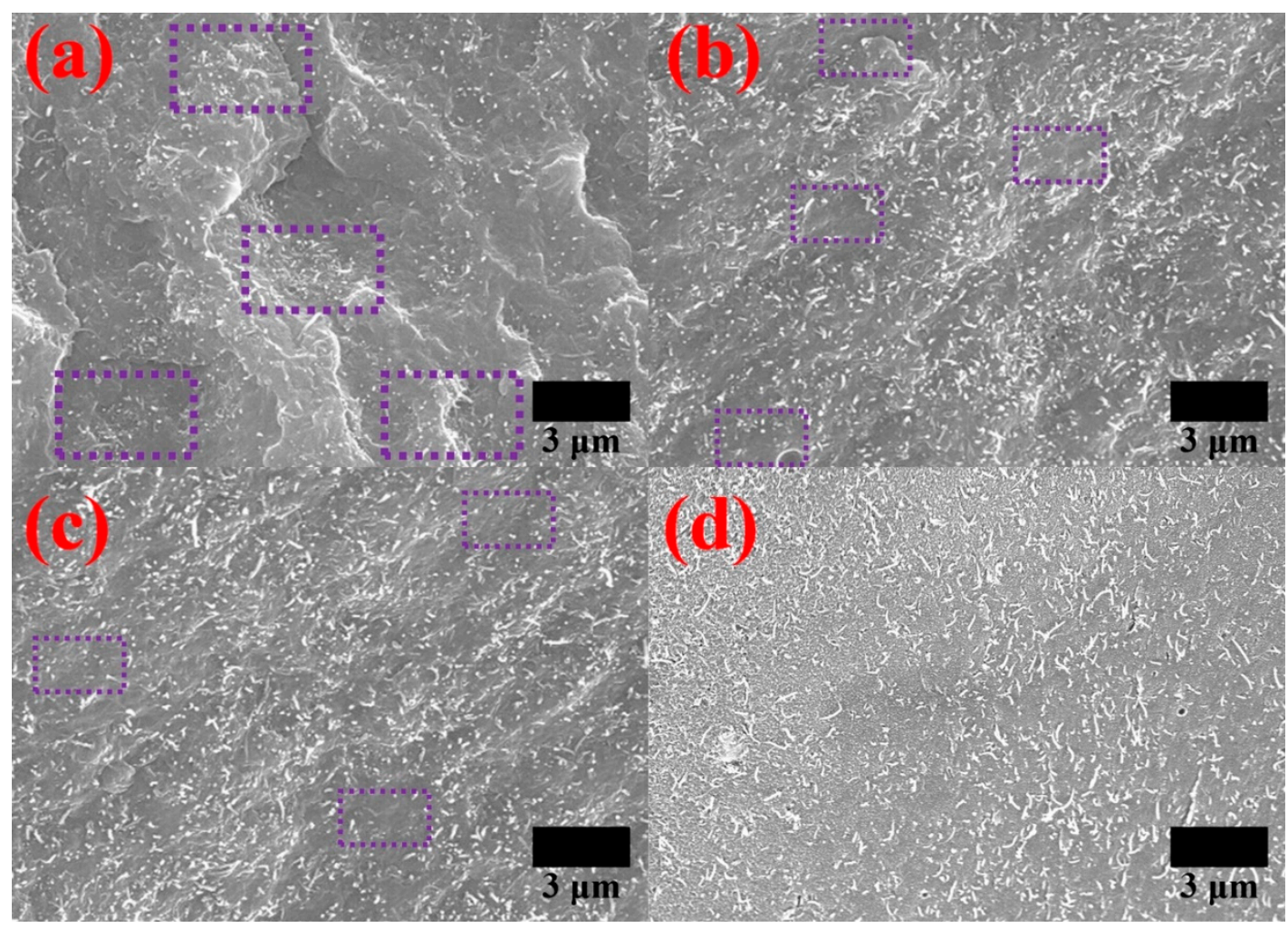

Figure 5. FE-SEM images of the composites filled with (a) purified MWCNTs, (b) carboxylated MWCNTs, (c) isocyanated MWCNTs, and (d) PA6-grafted MWCNTs.

\subsection{Tensile Properties of Composites}

The tensile properties of PA6 composites filled with $1 \mathrm{wt} \%$ MWCNTs are shown in Figure 6, and those of the PA6-grafted MWCNTs filled PA6 composites showed the highest results. The tensile strength and modulus of the PA6-grafted MWCNTs filled PA6 composites were increased by 25\% and $171 \%$, respectively, as compared to those of neat PA6. Further, they were $5.3 \%$ and $20.5 \%$ higher than those of the purified MWCNT-filled PA6, respectively. These improved tensile properties can be attributed to the improved dispersion of the PA6-grafted MWCNT in PA6 composites and the enhanced interfacial properties between the PA6-grafted MWCNT and PA6 matrix. In the case of the PA6 composites filled with the PA6-grafted MWCNT, because the surface of the MWCNT is covalently bonded to PA6 polymers, interdiffusion between the PA6 chain on the MWCNT surface and the PA6 matrix could occur, and the dispersion of the MWCNT in the composite was improved. In addition, amide exchange reactions can occur due to the high processing temperature, and the interfacial properties between them can be improved [38]. In the case of carboxylated and isocyanated MWCNT-filled PA6 composites, the tensile properties were confirmed to be superior to those of the purified MWCNT-filled PA6 composite, and this can be attributed to the hydrogen bonding between the functional groups in the MWCNTs and the PA matrix [39]. 

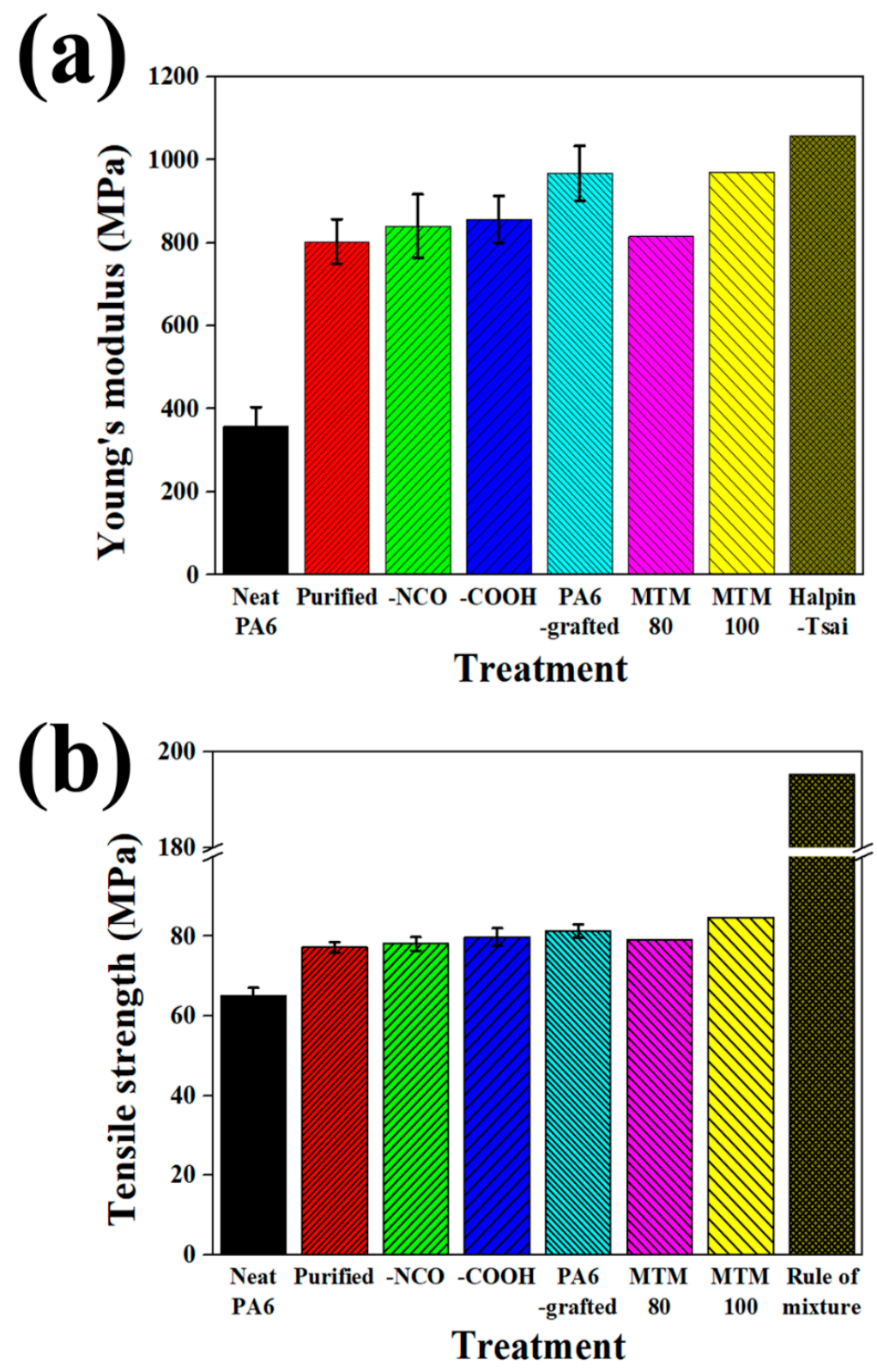

Figure 6. Measured and calculated tensile (a) modulus and (b) strength of PA6 composites.

Theoretically obtained tensile properties of the PA6 composites filled with $1 \mathrm{wt} \%$ MWCNTs are shown in Figure 6. The theoretical results were most similar to the actual results using PA6-grafted MWCNTs, due to their excellent dispersion and interfacial properties. Regarding tensile modulus, when the experimental result of the composite incorporating the PA6-grafted MWCNTs was compared with the theoretical results based on the classical Halpin-Tsai model, assuming the random orientation of MWCNTs (see Supplementary Materials) and based on the MTM assuming a perfect interface, the experimental result was $8.6 \%$ and $0.4 \%$ lower than the theoretical results, respectively. In the case of tensile strength, as compared with the theoretical results based on the rule of mixture (see Supplementary Materials) and based on the MTM assuming a perfect interface, the measured result was $58.4 \%$ and $4.0 \%$ lower than the theoretical results, respectively. These results indicated that more realistic effective tensile modulus can be derived by considering the MWCNT shape in the MTM, and that it was necessary to consider the MWCNT shape in the theoretical calculation in order to obtain the effective tensile strength corresponding to the actual value. By applying weakened interfacial properties to the MTM, we could simulate the tensile properties of PA6 composites containing $1 \mathrm{wt} \%$ MWCNTs according to the surface treatments. The tensile strength and modulus of the PA6 composites 
incorporating carboxylated and isocyanated MWCNTs can be calculated by applying the $80 \%$ level of interface, while those of the composites incorporating PA6-grafted MWCNT could be simulated by assuming a perfect interface, indicating that PA6 grafting can induce better interfacial properties.

\section{Conclusions}

The tensile properties of MWCNT-filled PA6 composites were optimized by carboxylated, isocyanated, and PA6-grafted functionalizations, as well as reactive extrusion based on the anionic polymerization of CL. Based on the FTIR results, it was confirmed that the functionalization strategy was successfully achieved. The optimum process conditions were derived by analyzing the relationship between the reactive extrusion processing parameters and the resultant molecular weight. The composites filled with the PA6-grafted MWCNTs showed the highest tensile properties because of their uniform MWCNT dispersion and excellent interfacial property. By comparing between the measured tensile properties and the theoretically calculated ones based on the modified MTM, the interfacial property between the PA6-grafted MWCNT and PA6 matrix was evaluated to be an almost perfect interface.

Supplementary Materials: The following are available online at http://www.mdpi.com/2073-4360/12/5/997/s1, Figure S1: Anionic polymerization of $\varepsilon$-caprolactam, Figure S2: Screw configuration and barrel temperature.

Author Contributions: Conceptualization, M.P. and S.Y.K.; methodology, J.Y.; validation, J.Y.; formal analysis, J.Y.; investigation, J.H.P.; writing — original draft preparation, M.P.; writing—-review and editing, S.Y.K.; visualization, J.-u.J.; supervision, S.Y.K.; project administration, M.P. All authors have read and agreed to the published version of the manuscript.

Funding: This work was supported by a grant from the Fundamental R\&D Program for Technology of World Premier Materials funded by the Ministry of Trade, Industry, and Energy, Republic of Korea. We acknowledge the financial support from a Korea Institute of Science and Technology internal project. This research was also supported by Basic Science Research Program (2017R1C1B5077037) through the National Research Foundation of Korea (NRF) funded by the Ministry of Education and the Ministry of Trade, Industry and Energy (MOTIE, Korea) under Industrial Technology Innovation Program. No. 10082586.

Conflicts of Interest: The authors declare no conflict of interest.

\section{References}

1. Iijima, S. Helical microtubules of graphitic carbon. Nature 1991, 354, 56-58. [CrossRef]

2. Treacy, M.M.J.; Ebbesen, T.W.; Gibson, J.M. Exceptionally high Young's modulus observed for individual carbon nanotubes. Nature 1996, 381, 678-680. [CrossRef]

3. Wong, E.W.; Sheehan, P.E.; Lieber, C.M. Nanobeam mechanics: Elasticity, strength, and toughness of nanorods and nanotubes. Science 1997, 277, 1971-1975. [CrossRef]

4. Spitalsky, Z.; Tasis, D.; Papagelis, K.; Galiotis, C. Carbon nanotube-polymer composites: Chemistry, processing, mechanical and electrical properties. Prog. Polym. Sci. 2010, 35, 357-401. [CrossRef]

5. Tjong, S.C. Structural and mechanical properties of polymer nanocomposites. Mater. Sci. Eng. R Rep. 2006, 53, 73-197. [CrossRef]

6. Roy, N.; Sengupta, R.; Bhowmick, A.K. Modifications of carbon for polymer composites and nanocomposites. Prog. Polym. Sci. 2012, 37, 781-819. [CrossRef]

7. Jang, J.-U.; Lee, H.S.; Kim, J.W.; Kim, S.Y.; Kim, S.H.; Hwang, I.; Kang, B.J.; Kang, M.K. Facile and cost-effective strategy for fabrication of polyamide 6 wrapped multi-walled carbon nanotube via anionic melt polymerization of $\varepsilon$-caprolactam. Chem. Eng. J. 2019, 373, 251-258. [CrossRef]

8. Wang, H.; Li, N.; Xu, Z.; Tian, X.; Mai, W.; Li, J.; Chen, C.; Chen, L.; Fu, H.; Zhang, X. Enhanced sheet-sheet welding and interfacial wettability of $3 \mathrm{D}$ graphene networks as radiation protection in gamma-irradiated epoxy composites. Compos. Sci. Technol. 2018, 157, 57-66. [CrossRef]

9. Zhbanov, A.I.; Pogorelov, E.G.; Chang, Y.-C. Van der waals interaction between two crossed carbon nanotubes. ACS Nano 2010, 4, 5937-5945. [CrossRef]

10. Li, W.; Shi, C.; Shan, M.; Guo, Q.; Xu, Z.; Wang, Z.; Yang, C.; Mai, W.; Niu, J. Influence of silanized low-dimensional carbon nanofillers on mechanical, thermomechanical, and crystallization behaviors of poly(L-lactic acid) composites-A comparative study. J. Appl. Polym. Sci. 2013, 130, 1194-1202. [CrossRef] 
11. Sahoo, N.G.; Rana, S.; Cho, J.W.; Li, L.; Chan, S.H. Polymer nanocomposites based on functionalized carbon nanotubes. Prog. Polym. Sci. 2010, 35, 837-867. [CrossRef]

12. Chen, J.; Yan, L.; Song, W.; Xu, D. Interfacial characteristics of carbon nanotube-polymer composites: A review. Compos. Part A Appl. Sci. Manuf. 2018, 114, 149-169. [CrossRef]

13. Park, M.; Lee, H.; Jang, J.-U.; Park, J.H.; Kim, C.H.; Kim, S.Y.; Kim, J. Phenyl glycidyl ether as an effective noncovalent functionalization agent for multiwalled carbon nanotube reinforced polyamide 6 nanocomposite fibers. Compos. Sci. Technol. 2019, 177, 96-102. [CrossRef]

14. Noh, Y.J.; Pak, S.Y.; Hwang, S.H.; Hwang, J.Y.; Kim, S.Y. Enhanced dispersion for electrical percolation behavior of multi-walled carbon nanotubes in polymer nanocomposites using simple powder mixing and in situ polymerization with surface treatment of the fillers. Compos. Sci. Technol. 2013, 89, 29-37. [CrossRef]

15. Pak, S.Y.; Kim, H.M.; Kim, S.Y.; Youn, J.R. Synergistic improvement of thermal conductivity of thermoplastic composites with mixed boron nitride and multi-walled carbon nanotube fillers. Carbon 2012, 50, 4830-4838. [CrossRef]

16. Yang, M.; Gao, Y.; Li, H.; Adronov, A. Functionalization of multiwalled carbon nanotubes with polyamide 6 by anionic ring-opening polymerization. Carbon 2007, 45, 2327-2333. [CrossRef]

17. Yuen, S.-M.; Ma, C.-C.M.; Lin, Y.-Y.; Kuan, H.-C. Preparation, morphology and properties of acid and amine modified multiwalled carbon nanotube/polyimide composite. Compos. Sci. Technol. 2007, 67, 2564-2573. [CrossRef]

18. Gao, J.; Itkis, M.E.; Yu, A.; Bekyarova, E.; Zhao, B.; Haddon, R.C. Continuous spinning of a single-walled carbon nanotube-nylon composite fiber. J. Am. Chem. Soc. 2005, 127, 3847-3854. [CrossRef]

19. Li, W.; Xu, Z.; Chen, L.; Shan, M.; Tian, X.; Yang, C.; Lv, H.; Qian, X. A facile method to produce graphene oxide-g-poly(L-lactic acid) as an promising reinforcement for PLLA nanocomposites. Chem. Eng. J. 2014, 237, 291-299. [CrossRef]

20. Chen, L.; Li, X.; Wang, L.; Wang, W.; Xu, Z. Effect of the molecular chains grafted on graphene nanosheets on the properties of poly(l-lactic acid) nanocomposites. Polym. Compos. 2015, 38, 5-12. [CrossRef]

21. Kim, H.S.; Jang, J.-U.; Lee, H.; Kim, S.Y.; Kim, S.H.; Kim, J.; Jung, Y.C.; Yang, B.J. Thermal management in polymer composites: A review of physical and structural parameters. Adv. Eng. Mater. 2018, 20, 1800204. [CrossRef]

22. Noh, Y.J.; Joh, H.-I.; Yu, J.; Hwang, S.H.; Lee, S.; Lee, C.H.; Kim, S.Y.; Youn, J.R. Ultra-high dispersion of graphene in polymer composite via solvent free fabrication and functionalization. Sci. Rep. 2015, 5, 9141. [CrossRef] [PubMed]

23. Ni, Y.; Chen, L.; Teng, K.; Shi, J.; Qian, X.; Xu, Z.; Tian, X.; Hu, C.; Ma, M. Superior mechanical properties of epoxy composites reinforced by 3D interconnected graphene skeleton. ACS Appl. Mater. Interfaces 2015, 7, 11583-11591. [CrossRef] [PubMed]

24. Kim, H.S.; Kim, J.H.; Yang, C.-M.; Kim, S.Y. Synergistic enhancement of thermal conductivity in composites filled with expanded graphite and multi-walled carbon nanotube fillers via melt-compounding based on polymerizable low-viscosity oligomer matrix. J. Alloy. Compd. 2017, 690, 274-280. [CrossRef]

25. Kim, S.Y.; Noh, Y.J.; Yu, J. Improved thermal conductivity of polymeric composites fabricated by solvent-free processing for the enhanced dispersion of nanofillers and a theoretical approach for composites containing multiple heterogeneities and geometrized nanofillers. Compos. Sci. Technol. 2014, 101, 79-85. [CrossRef]

26. Kim, S.Y.; Noh, Y.J.; Yu, J. Prediction and experimental validation of electrical percolation by applying a modified micromechanics model considering multiple heterogeneous inclusions. Compos. Sci. Technol. 2015, 106, 156-162. [CrossRef]

27. Kim, S.Y.; Noh, Y.J.; Yu, J. Thermal conductivity of graphene nanoplatelets filled composites fabricated by solvent-free processing for the excellent filler dispersion and a theoretical approach for the composites containing the geometrized fillers. Compos. Part A Appl. Sci. Manuf. 2015, 69, 219-225. [CrossRef]

28. Noh, Y.J.; Kim, S.Y. Synergistic improvement of thermal conductivity in polymer composites filled with pitch based carbon fiber and graphene nanoplatelets. Polym. Test. 2015, 45, 132-138. [CrossRef]

29. Noh, Y.J.; Kim, H.S.; Ku, B.-C.; Khil, M.-S.; Kim, S.Y. Thermal conductivity of polymer composites with geometric characteristics of carbon allotropes. Adv. Eng. Mater. 2016, 18, 1127-1132. [CrossRef]

30. Yu, J.; Cha, J.E.; Kim, S.Y. Thermally conductive composite film filled with highly dispersed graphene nanoplatelets via solvent-free one-step fabrication. Compos. Part B Eng. 2017, 110, 171-177. [CrossRef] 
31. Yu, J.; Lacy, T.E.; Toghiani, H.; Pittman, C.U. Classical micromechanics modeling of nanocomposites with carbon nanofibers and interphase. J. Compos. Mater. 2011, 45, 2401-2413. [CrossRef]

32. Jung, H.; Choi, H.K.; Yu, J. Prediction and experimental validation of composite strength by applying modified micromechanics for composites containing multiple distinct heterogeneities. Compos. Part B Eng. 2016, 91, 1-7. [CrossRef]

33. Davé, R.S.; Kruse, R.L.; Stebbins, L.R.; Udipi, K. Polyamides from lactams via anionic ring-opening polymerization: 2. Kinetics. Polymer 1997, 38, 939-947. [CrossRef]

34. Ricco, L.; Russo, S.; Orefice, G.; Riva, F. Anionic poly( $\varepsilon$-caprolactam): relationships among conditions of synthesis, chain regularity, reticular order, and polymorphism. Macromolecules 1999, 32, 7726-7731. [CrossRef]

35. Wittmer, V.P.; Gerrens, H. Über die anionische schnellpolymerisation von caprolactam. Macromol. Chem. Phys. 1965, 89, 27-43. [CrossRef]

36. Zhang, W.D.; Shen, L.; Phang, I.Y.; Liu, T. Carbon nanotubes reinforced nylon-6 composite prepared by simple melt-compounding. Macromolecules 2004, 37, 256-259. [CrossRef]

37. Fornes, T.D.; Yoon, P.J.; Keskkula, H.; Paul, D.R. Nylon 6 nanocomposites: The effect of matrix molecular weight. Polymer 2001, 42, 09929-09940. [CrossRef]

38. Koning, C.; Duin, M.V.; Pagnoulle, C.; Jerome, R. Strategies for compatibilization of polymer blends. Prog. Polym. Sci. 1998, 23, 707-757. [CrossRef]

39. Liu, T.; Phang, I.Y.; Shen, L.; Chow, S.Y.; Zhang, W.D. Morphology and mechanical properties of multiwalled carbon nanotubes reinforced nylon-6 composites. Macromolecules 2004, 37, 7214-7222. [CrossRef]

(C) 2020 by the authors. Licensee MDPI, Basel, Switzerland. This article is an open access article distributed under the terms and conditions of the Creative Commons Attribution (CC BY) license (http://creativecommons.org/licenses/by/4.0/). 